\title{
Multi-Image Studies of Atypical Location of Ventricular Aneurysms and Survival: Clinical Cases Series
}

\author{
Gyselle Camacho-Camacho', Isabel Carvajal-Juárez', Hector Guatibonza-Zambrano², \\ Alejandra de la Torre², Aloha Meave-Gonzalez², Candace Keirns ${ }^{3}$, Erick Alexanderson-Rosas ${ }^{1,4}$, \\ Alondra Flores-García ${ }^{1}$, Nilda Espinola-Zavaleta ${ }^{1,5^{*}}$
}

\author{
${ }^{1}$ Department of Nuclear Cardiology, National Institute of Cardiology Ignacio Chavez, Mexico City, Mexico \\ ${ }^{2}$ Department of Resonance Imaging, National Institute of Cardiology Ignacio Chavez, Mexico City, Mexico \\ ${ }^{3}$ International Medical Interpreters Association, Boston, ME, USA \\ ${ }^{4}$ Department of Physiology, School of Medicine, UNAM, Mexico City, Mexico \\ ${ }^{5}$ Department of Echocardiography, ABC Medical Center, I.A.P., Mexico City, Mexico \\ Email: giizcamacho@gmail.com, estelais.md@gmail.com, hazambrano2000@gmail.com, alejandra.delatorreg@gmail.com, \\ ameaveg@gmail.com,mieshe@comcast.net, alexandersonerick@gmail.com, alondra.floresg@gmail.com, *niesza2001@hotmail.com
}

How to cite this paper: Camacho-Camacho, G., Carvajal-Juárez, I., Guatibonza-Zambrano, H., de la Torre, A., Meave-Gonzalez, A., Keirns, C., Alexanderson-Rosas, E., Flores-García, A. and Espinola-Zavaleta, N. (2019) Multi-Image Studies of Atypical Location of Ventricular Aneurysms and Survival: Clinical Cases Series. World Journal of Cardiovascular Diseases, 9, 524-537.

https://doi.org/10.4236/wjcd.2019.98045

Received: July 4, 2019

Accepted: August 10, 2019

Published: August 13, 2019

Copyright $\odot 2019$ by author(s) and Scientific Research Publishing Inc. This work is licensed under the Creative Commons Attribution International License (CC BY 4.0).

http://creativecommons.org/licenses/by/4.0/

(c) (i) Open Access

\begin{abstract}
Background: A left ventricular aneurysm is a mechanical complication of a myocardial infarction that frequently develops on the anterior and apical wall. Survival of 3 to 5 years is $27 \%$ and $12 \%$, respectively. Our objective is to report 3 cases of ventricular aneurysms in atypical locations and analyze the survival of these patients. Material and Methods: Three patients with suspicion of ventricular aneurysm after acute myocardial infarction who sought attention at our institution were included. All underwent transthoracic echocardiograms (TTE), cardiac magnetic resonance (CMR), nuclear cardiology (NC), coronary angiotomography and cardiac catheterization to assess the location and characteristics of the aneurysms, left ventricular systolic function and the anatomical and functional condition of the coronary arteries. Results: The mean age of studied patients was $58.33 \pm 10.37$ years. The locations of the ventricular aneurysms were lateral, inferior and septal. The patients received optimal medical treatment to control heart failure and were discharged in stable condition without early mortality. The mean follow-up was 6.33 years (intervals: 2 - 9) and during this period the patients were in NYHA functional class I/II. Conclusion: Left ventricular aneurysm is a late mechanical complication of an infarction that can develop in an atypical location. Diagnosis is achieved using non-invasive techniques such as TTE, CMR, NC, and coronary angiotomography. No mortality occurred among the patients during medium and long-term follow-up.
\end{abstract}




\section{Keywords}

Aneurysm, Myocardial Infarction, Echocardiography, Cardiac Magnetic Resonance, Coronary Angiogram, Survival

\section{Introduction}

Left ventricular aneurysm (LVA) is a late complication of a myocardial infarction. It is defined as an area of the ventricular myocardium with a thinner myocardium of the adjacent wall that manifests akinesia or dyskinesia and that affects the entire thickness of the wall. The location of an LVA in up to $80 \%$ of cases is the antero-apical wall associated with obstruction of anterior descending artery [1]. Survival to 3 and 5 years is $27 \%$ and $12 \%$, respectively, with appropriate surgical treatment survival to 5 years increases to $71 \%$ [2].

Less common causes of aneurysms include trauma, hypertrophic or congenital cardiomyopathy, infective endocarditis, inflammatory disease such as Chagas disease or sarcoidosis and idiopathic dilated cardiomyopathy [3].

The principal symptoms are dyspnea, angina, and ventricular arrhythmias. Complications include systemic embolism and rupture of the left ventricular wall [4]. Transthoracic echocardiography is the diagnostic technique of choice for LVA, although transesophageal echocardiography, left ventriculography, cardiac computed tomography and CMR have higher sensitivity [5] [6] [7] [8].

The objective of this study was to present three patients with myocardial infarction who were selected because they developed atypical aneurysms and had a greater survival than the described in the literature.

\section{Material and Methods}

Three patients with LVA in atypical locations who attended the Ignacio Chavez National Institute of Cardiology in the period from August 2018 to June 2019 were included in our study. All patients had complete clinical histories and non-invasive imaging studies including transthoracic echocardiography (TTE), cardiac magnetic resonance (CMR) and nuclear cardiology (NC) performed. They also underwent cardiac catheterization.

The study periods and study site were advised to be provided in the Materials and methods section.

\subsection{Echocardiography}

Two and three-dimensional transthoracic echocardiograms were performed on all patients using a Siemens Acuson 2000 machine with a mechanical probe with M-mode, color and tissue Doppler and 2D and 3D capabilities. The patient assumed a lateral decubitus position. Measurements of heart chambers and wall thickness were taken from parasternal long axis images in systole and diastole. Global and segmental mobility of both ventricles was assessed from parasternal 
short axis images from the ventricular base to the apex. The ejection fraction was calculated from apical 4 and 2 chamber images by a modified Simpson method according to the guidelines of the American Society of Echocardiography and the European Society of Echocardiography [5]. Doppler color was used to evaluate the presence and severity of valvular regurgitation, while the systolic pressure of the pulmonary artery was calculated with color and continuous wave Doppler using the tricuspid regurgitation method with Bernoulli's equation and the right atrial pressure taking into account the diameter and collapse of the inferior vena cava. The left ventricular beat-to-beat ejection fraction was calculated using the matrix $3 \mathrm{D}$ probe.

\subsection{Cardiac Magnetic Resonance}

CMR imaging was performed using a 1.5-T magnetic resonance imaging system (Avanto Fit; Siemens, Erlangen, Germany) with a phased-array body coil and electrocardiogram (ECG) monitoring. The imaging protocol consisted of the following sequences: 1) scout images, 2) T1-weighted imaging, 3) T2-weighted imaging, 4) cine 2D steady-state free precession (SSFP) imaging of the left ventricle $(\mathrm{LV}), 5)$ dynamic first-pass contrast enhancement perfusion imaging in representative short-axis planes, and 6) inversion-recovery late gadolinium enhancement imaging 5 and 10 minutes after intravenous injection of a total of $0.01 \mathrm{mmol} / \mathrm{kg}$ gadobutrol (Gadovist $1 \mathrm{mmol} / \mathrm{ml}$; Bayer HealthCare). Imaging was performed during repeated end expiratory breath-holds to minimize respiratory motion artifact [6].

\subsection{Nuclear Cardiology}

Myocardial perfusion was evaluated in a resting state with $370 \mathrm{MBq}$ of ${ }^{99 \mathrm{~m}} \mathrm{Tc}-\mathrm{MIBI}$ and $740 \mathrm{MBq}$ under stress. The stress phase was performed with physical exercise (Bruce protocol) or with dipyridamole (dose $0.56 \mathrm{mg} / \mathrm{kg}$ ) based on the clinical characteristics of each patient. Static and gated images were acquired on a Symbia Siemens ${ }^{\circledR}$ gammacamera with a Smart-zoom collimator (cardiocentric orbit). Resting images were acquired with a $128 \times 128$ matrix, Zoom1.0, $20 \%$ the energetic window centered at $140 \mathrm{keV}$, with 14 seconds per image (16 frames). Stress images were acquired with the same parameters except with 9 seconds per image (16 frames) [7].

\subsection{Cardiac Catheterization}

This study was performed in an experimented hemodynamic service by expert cardiologists following previously described guidelines [8] [9].

\section{Results}

The mean age of studied patients was $58.33 \pm 10.37$ years. The locations of the ventricular aneurysms were lateral, inferior and septal, respectively. The patients received optimal medical treatment to control heart failure and were discharged in 
stable condition without early mortality. The mean follow-up was of 6.33 years (intervals: 2 - 9) and during this period the patients were NYHA functional class I/II.

\subsection{Case 1}

This patient was a 51-year-old male with a heart murmur detected at 3 months of age. The ECG showed sinus rhythm and a hemiblock of the anterior division of the left branch of the His bundle. He was treated with digitalis from 2 to 3 years of age. Two cardiac catheterizations were performed when he was 5 and 12 years old, without pathologic results. In 2009 at 42 years of age he suffered a transitory cerebral ischemic attack characterized by aphasia, dizziness, left hemianopsia and intense headache with spontaneous resolution. Because of this event he sought attention in the emergency department of our institution for evaluation. The EKG showed sinus rhythm and rectification of the ST segment in all the leads of the left ventricle and an $\mathrm{S}$ wave from $\mathrm{V}_{1}$ to $\mathrm{V}_{6}$. The TTE revealed an aneurysm of the lateral wall in the basal and middle portions with severe systolic dysfunction and an ejection fraction (LVEF) of 30\%, dilated right chambers and mild pulmonary hypertension with a PSAP of $38 \mathrm{mmHg}$.

CMR corroborated an aneurysm of the basal and middle portions of the lateral wall of the LV with a diameter of $59 \times 30 \mathrm{~mm}$ and a neck of $29 \mathrm{~mm}$ (clips 1, 2). Fibrosis was found in the aneurysmal area, and systolic dysfunction was severe with a LVEF of $27 \%$. Epicardial coronary arteries were normal on the coronary angiotomography and an aneurysm of the LV on the anterolateral and inferolateral wall of $65 \times 40 \mathrm{~mm}$ and severe left ventricular systolic dysfunction was detected. Cardiac catheterization showed epicardial arteries without significant obstruction and generalized hypokinesis of the LV with an anterolateral aneurismal area and LVEF of 28\% (Figure 1). The myocardial perfusion study reported a transmural infarction of the basal third of the anterolateral area that extended non-transmurally to the middle third and apical region with mild ischemia of the residual tissue suggesting the territory of the left anterior descending artery. A non-transmural infarction of the infero-septal region in the middle and apical thirds and transmural infarction in the basal third with mild ischemia of the residual tissue probably corresponding to the territory of the right coronary artery and moderate intraventricular asynchrony because of delayed contraction of the lateral aneurysmal area were observed. Chagas disease was ruled out as the origin of the disease.

In 2011, biventricular pacing was implanted to correct bigeminy and trigeminy, and he continued medical treatment with aspirin, metoprolol, amiodarone, digoxin, atorvastatin, enalapril, spironolactone and acenocoumarol.

The 2018 control echocardiogram and myocardial perfusion study demonstrated improved left ventricular systolic function with a LVEF of $40 \%$ and dyssynchrony (Figure 2).

Over the approximately 8 years of follow-up the patient has remained in NYHA functional class II. 




Figure 1. Case 1. (A) Resting ECG in sinus rhythm, HR $64 \mathrm{bpm}, \mathrm{LBBB}$ and rectification of the ST segment from $V_{1}$ to $V_{6}$. (B) Transthoracic echocardiographic apical 4 chamber image that shows an aneurysm of the basal and middle portions of the left ventricular lateral wall (white arrows); (C) Cardiac magnetic resonance on an apical 4 chamber plane with an aneurysm of the basal and middle portions of the lateral wall (clips 1 and 2); (D) Late gadolinium enhanced image showing continuous transmural enhancement (white arrows) in the aneurismal area without pericardial enhancement; (E) Coronary angiogram shows the right coronary artery without obstructive lesions; (F) Left coronary artery with no obstructions. LV: Left ventricle, LA: Left atrium, RV: Right ventricle, RA: Right atrium.

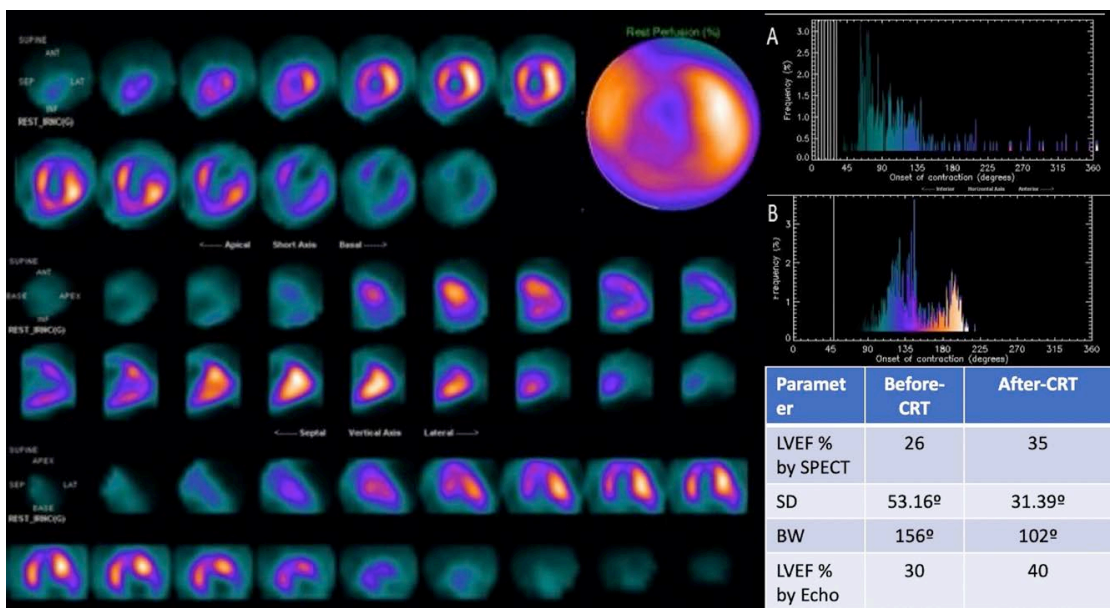

Figure 2. Case 1. SPECT-Myocardial perfusion, synchrony and systolic function. A transmural infarction of the basal third of the anterolateral area that extended non-transmurally to the middle third and apical region with mild ischemia of the residual tissue was found, suggesting the territory of the left anterior descending artery. A non-transmural infarction of the infero-septal region in the middle and apical thirds and transmural infarction in the basal third with mild ischemia of the residual tissue corresponding to the territory of the right coronary artery. (A) Standard deviation, bandwidth and systolic function before and (B) after cardiac resynchronization therapy.

\subsection{Case 2}

This 51-year-old man had a history of type 2 diabetes mellitus (DM), systemic 
hypertension (HT), an acute myocardial infarction (AMI) without revascularization and without a specified location in 2010 and an AMI without ST segment elevation in 2016. Coronary angiography revealed a circumflex artery with ectasia in the proximal segment, collateral circulation to the posterior descending artery, a $60 \%$ obstruction in the proximal segment of the right coronary artery, a $30 \%$ obstruction in the final portion of the proximal segment and total occlusion of the vertical segment. The patient was treated with acetylsalicylic acid, clopidogrel, atorvastatin, isosorbide dinitrate, amiodarone, metformin, metoprolol and enalapril. The patient assisted to the emergency department of our institution with oppresive precordial pain of $4 / 10$ intensity with palpitations, nausea and vomiting. The EKG showed sustained monomorphic ventricular tachycardia that reverted to sinus rhythm with electric cardioversion (150 Joules). Dilatation of left heart chambers, an aneurysm of the basal and middle portions of left ventricular inferior wall and severe left ventricular systolic dysfunction with an LVEF of $27 \%$ were detected by TTE. The myocardial perfusion study demonstrated a LV transmural infero-septal, inferior and basal and middle infero-lateral infarction involving the right ventricle, infero-septal dyskinesia in the basal and middle thirds, biventricular systolic dysfunction with a LVEF of 33\%, a RVEF of $30 \%$ and mild dilatation of the left ventricle.

The patient was discharged with clinical improvement and treatment based on acetylsalicylic acid, atorvastatin, amiodarone, carvedilol, enalapril, trimetazidine, spironolactone, levothyroxine and acenocoumarin.

In 2018, CMR showed an infarction in the inferior wall and infero-septal region that was transmural in the basal third and not transmural in the middle and apical thirds, infero-septal dyskinesia in the basal and middle third and improved systolic function with a LVEF of $45 \%$, clip 3. Control TTE demonstrated an inferior aneurysm affecting the basal and middle segments and LVEF of $45 \%$ (Figure 3).

During the 9 years of follow-up the patients has remained in NYHA functional class I.

\subsection{Case 3}

This 72-year-old female had a history of HT and type 2 diabetes. She came to the emergency department of her local hospital with oppressive chest pain that spread to her neck and back of 45 minutes duration with an intensity of 10/10 and shortness of breath. Treatment was begun with aspirin and atorvastatin. Nine days later she started with episodes of precordial pain with moderate efforts and shortness of breath so she attended the emergency department of our institution. The EKG revealed sinus rhythm, elevation of the ST segment from $\mathrm{V}_{2}$ to $\mathrm{V}_{6}$, inverted $\mathrm{T}$ waves in the remaining leads and $\mathrm{Q}$ waves in $A V F$ and $\mathrm{D}_{\mathrm{III}}$. The NT-proBNP levels were $6872 \mathrm{pg} / \mathrm{ml}$. TTE showed dilatation of left chambers, lateral aneurysm in its apical portion and septal apical pseudoaneurysm with laminar thrombus, left ventricular systolic dysfunction with a LVEF of $38 \%$, 


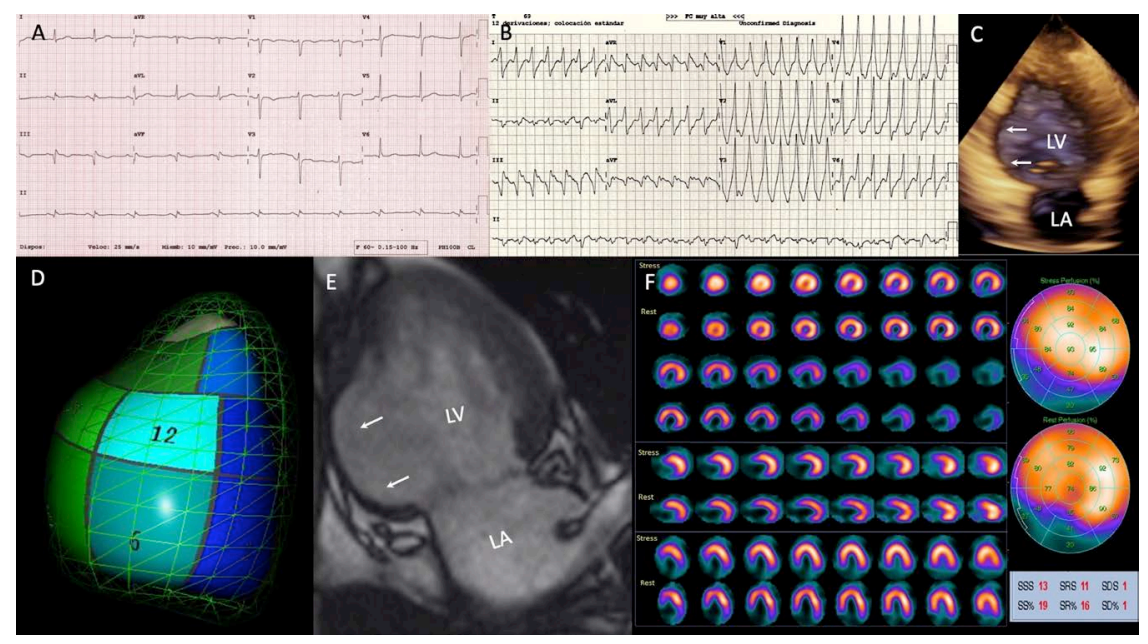

Figure 3. Case 2. (A) Resting ECG in sinus rhythm, HR $75 \mathrm{bpm}$, Q wave in $\mathrm{D}_{\mathrm{II}}, \mathrm{D}_{\mathrm{III}}$, and AVF; (B) Monomorphic sustained ventricular tachycardia. (C) and (D) 3D transthoracic echocardiogram in the apical 2-chamber showing aneurysm of the basal and middle portions of the left ventricular inferior wall (white arrows); (E) Cardiac magnetic resonance that demonstrates the aneurysm in the basal and middle portions of the left ventricular inferior wall (white arrows), clip 3; (F) Myocardial perfusion study shows the transmural infarction of the inferior wall in its basal portion that extends non-transmurally to the middle third and to infero-septal and infero-lateral segments with mild infero-lateral basal ischemia. LV: Left ventricle, LA: Left atrium.

moderate mitral regurgitation, mild tricuspid regurgitation and pulmonary hypertension with PSAP of $42 \mathrm{mmHg}$.

CMR confirmed the septal pseudoaneurysm in its apical portion containing a thrombus, lateral aneurysm, left ventricular systolic dysfunction with LVEF of $38 \%$, normal right ventricular systolic function with RVEF of $69 \%$, moderate mitral regurgitation and mild tricuspid regurgitation, clip 4.

The myocardial perfusion study demonstrated a transmural infarction of the apex and apical third of the antero-septal region and anterior wall without residual ischemic tissue, mild ischemia of the apical third of the infero-septal region and inferior wall, moderate dilatation of the left ventricle, generalized hypokinesis of the left ventricle with akinesis of the apex and severe systolic dysfunction with intraventricular asynchrony assessed by standard deviation and bandwidth (Figure 4). The coronary angiogram reported an eccentric tubular lesion of the left anterior descending artery with regular borders and $60 \%$ stenosis of the proximal segment and an eccentric tubular lesion with regular borders and $80 \%$ stenosis in the middle segment; the dominant circumflex artery had a short eccentric lesion with regular borders with 50\% stenosis in the distal segment (Figure 5). The patient rejected the surgery, so she was discharged with medical treatment based on aspirin, acenocoumarol, atorvastatin, captopril, metoprolol, isosorbide and metformin. During the 2-year follow-up, the patient remained in NYHA functional class II.

The three patients with ischemic heart disease who developed ventricular aneurysms with atypical locations were diagnosed by imaging studies, including TTE, 


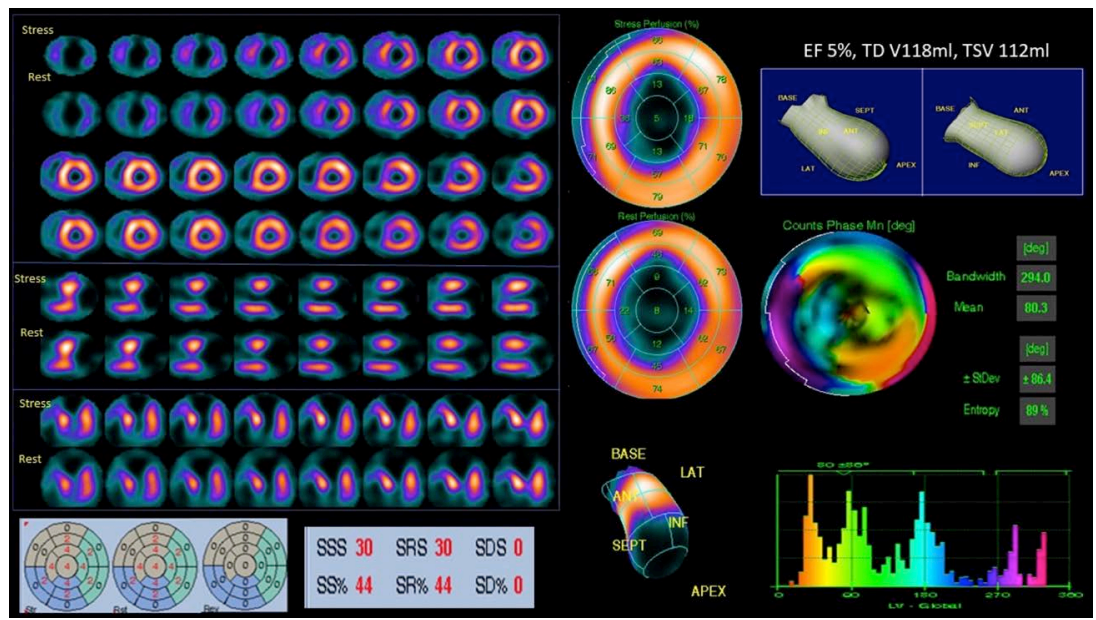

Figure 4. Case 3. Myocardial perfusion study SPECT that shows a transmural infarction of the apex and apical third of the antero-septal region and anterior wall without ischemia of the residual tissue, mild ischemia of the apical third of the infero-septal region. Intraventricular asynchrony was also observed.



Figure 5. Case 3. (A) Resting ECG in sinus rhythm, HR $70 \mathrm{bpm}$. Poor progression of the $r$ from $V_{1}$ to $V_{5}$ con ST segment elevation and inversion of the $T$ wave from $V_{1}$ to $V_{6}$. (B) 2-D transthoracic echocardiogram and color Doppler (C), that shows a pseudoaneurysm of the apical portion of the interventricular septum with a thrombus within it (arrow heads) and an aneurysm of the apical portion of the lateral wall (arrow) and mitral regurgitation. (D) the CMR corroborated the findings described in B, clip 4. (E) The fusion of SPECT/CMR showed uptake on left ventricular basal segments; noticed the absence of uptake on apex and apical segments correlated with the aneurysm and the pseudoaneurysm. (F) Coronary angiography shows an eccentric tubular lesion with regular borders and $60 \%$ stenosis in the proximal segment, an eccentric tubular lesion with regular borders and $80 \%$ stenosis in the middle segment of the anterior descending artery and a short eccentric lesion with regular borders and $50 \%$ stenosis in the distal segment of the circumflex artery. LV: Left ventricle, LA: Left atrium, RV: Right ventricle, RA: Right atrium, LAD: Left anterior descending artery, CX: Circumflex artery.

CMR, myocardial perfusion, coronary angiotomography and cardiac catheterization. The mean LVEF on admission was $29 \% \pm 0.5 \%$, while during follow-up (mean $6.5 \pm 3.9$ years) under optimal pharmacological treatment the LVEF improved to $41 \% \pm 3.6 \%$ (Figure 6 ). 


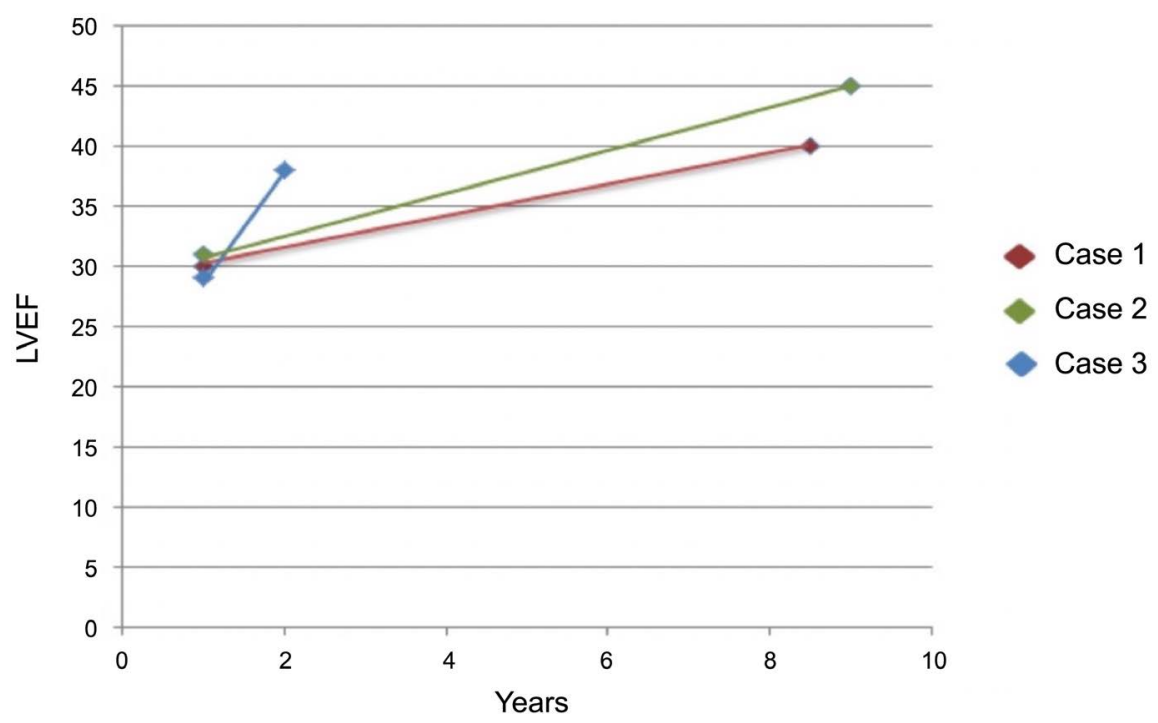

Figure 6. Improvement of left ventricular ejection fraction during follow-up. LVEF: Left ventricular ejection fraction.

\section{Discussion}

Ventricular aneurysms are late complications of myocardial infarctions. Extension of an anterior infarction occurs in 35 to $45 \%$ of cases and at lower percentages for infarctions in other locations-10\% - 15\% involving the inferior wall, $7.9 \%$ the interventricular septum and only $1 \%$ the lateral wall [10]. Ventricular aneurysms of the lateral wall correspond to obstruction of the anterior descending artery and the marginal branch of the circumflex coronary artery, while in cases involving the inferior wall are due to descending artery, right coronary and circumflex artery obstruction [11]. The formation of aneurysms increases mortality due to the complications they precipitate, such as ventricular arrhythmias, embolism and moderate to severe mitral regurgitation secondary to distortion of papillary muscle anatomy. When any of these complications exists revascularization of the affected coronary artery together with resection of the aneurysm and/or mitral valve repair should be performed. However, when surgical risk is high, anticoagulation and reduction of afterload is recommended [5].

A ventricular septal aneurysm is rarely detected in the adult population. Among pediatric patients it is associated with congenital heart disease in $0.3 \%$ and secondary to trauma or infection in other cases [12] [13]. There are some reports of patients between the ages of 30 and 50 who developed atypical aneurysms, which initially manifest neurological symptoms such as motor aphasia and unilateral hemiparesis, even before presenting cardiac symptoms such as chest pain and dyspnea in exertion [12], as it happened with the case 1. Septal aneurysms have been associated with eccentric flow that constantly hits the septum because of mitral stenosis [14].

In patients with myocardial infarction it is very important to distinguish between true aneurysms and pseudoaneurysms. LV pseudoaneurysm is a result of rupture of the ventricular free wall but contained by the adherent pericardium, 
organizing thrombus and hematoma, while the true LV aneurysm is an area of thinned myocardium that is dyskinetic and involves the full thickness of the wall [15].

In the literature, there is one case of 59-years-old female with cardiovascular risk factors who had anterior ST-elevation myocardial infarction. The noninvasive imaging techniques (echocardiography, MRI and CT) showed a large left ventricular pseudoaneurysm in the apical portion of the interventricular septum with laminar thrombus. The pseudoaneurysm was contained within the pericardial space. The coronary angiography revealed occlusion of mid left anterior descending artery (LAD) with otherwise unobstructed vessels. The coronary angioplasty of LAD was failed and the patient underwent urgent surgical resection of pseudoaneurysm with good results [16]. Our case 3 with aneurysm and pseudoaneurysm received only medical treatment. With recent advancements in cardiac surgery and interventional percutaneous techniques, perioperative mortality has reached $10 \%$ [17].

From a clinical point of view inferior and lateral aneurysms were the most difficult to diagnose; generally, the diagnosis required ventriculography [11]. Today the diagnosis of ventricular aneurysms is achieved with non-invasive imaging techniques. Initial assessment with transthoracic echocardiography may be unrevealing, but in suspected cases angiography, transesophageal echocardiography, myocardial perfusion, coronary angiotomography and CMR have a higher diagnostic yield [5] [6] [7] [8] [9] [18]. The high spatial resolution and tissue characterization of CMR make it ideal for evaluation of LV pseudoaneurysm and for distinguishing pseudoaneurysms from true aneurysms, specially when it is fused with myocardial perfusion study. The use of late gadolinium enhancement to identify the location and transmural extent of prior infarcts is particularly valuable (Table 1) [19]. Despite recent advances in multi-imaging, the perioperative evaluation of tissue layers remains the gold standard for the differential diagnosis of these two entities [4] [18].

LVEF is an important factor for survival of patients with this type of aneurysms. Patients with LVEF $>30 \%$ have been reported to have a 5 -year survival of $77 \%$, while patients with an LVEF $<30 \%$ have a probability of survival of $64 \%$ [9].

Table 1. Characteristics of CMR to differentiate between true aneurysm from pseudoaneurysm (Sharma, A., et al. Cardiovasc Diagn Ther. 2015; 5(6): 464-470).

\begin{tabular}{ccc}
\hline CMR features & Aneurysm & Pseudoaneurysm \\
\hline Anatomic localization & Apical or anterior & Posterior or inferior \\
Neck & Wide & Narrow \\
Neck/mounth ratio & $0.9-1$ & $0.25-0.5$ \\
Contractility & Dyskinesia & Akinesia \\
$\begin{array}{c}\text { Appearance in } \\
\text { viability imaging }\end{array}$ & Enhancement in the wall of sac & Enhancement of \\
\end{tabular}


For our patients, myocardial perfusion studies let us establish a relation between the location of the ventricular aneurysm and the affected artery. In the first case of a lateral aneurysm, the myocardial perfusion study revealed a transmural infarction that mainly affected the lateral wall. In Case 2, myocardial perfusion showed an infarction of the inferior wall and infero-septal region that was transmural in the basal third but not transmural in the middle and apical thirds, with infero-septal dyskinesia in the basal and middle thirds. Coronary angiography demonstrated obstructive lesions in the posterior descending, circumflex and right coronary arteries. In Case 3 the myocardial perfusion study showed a transmural septal infarction.

Cardiac catheterization is an invasive method that still remains essential in the diagnosis and treatment of coronary lesions.

All of our patients have demonstrated improvement in their functional class with pharmacological treatment. The mean LVEF during follow-up was $41 \%$, which indicates a good prognosis for survival in these patients.

\section{Conclusions}

The location of ventricular aneurysms varies according to the obstruction site in coronary arteries.

Non-invasive imaging techniques are extremely valuable in the diagnosis and follow-up of patients with ventricular aneurysms in atypical locations.

Surgical intervention is contemplated only for cases of failed medical treatment, hemodynamic instability, refractory heart failure or ventricular tachyarrhythmias.

Survival of these patients depends on the precise diagnosis and optimal treatment.

\section{Acknowledgements}

The authors would like to acknowledge all the entire staff of the Nuclear Cardiology Department at Instituto Nacional de Cardiologia Ignacio Chavez, particularly to the nurses and technicians.

\section{Ethics Approval and Consent to Participate}

All procedures performed in studies involving human participants were in accordance with the ethical standards of the institutional and/or national research committee and with the 1964 Helsinki declaration and its later amendments or comparable ethical standard. This study was approved by the Ethical Committee of our institution.

\section{Consent for Publication}

All patients included in this study signed the institutional consent form.

\section{Availability of Data and Material}

The datasets used and/or analysed during the current study are available from the corresponding author on reasonable request. 


\section{Authors' Contributions}

GCC: Research idea, study design, data acquisition, data analysis, statistical analysis, ICJ: Research idea, manuscript drafting and supervision. HGZ and AT: Interpretation of CMR images and manuscript drafting. AMG: Research idea and supervision. CK: translation into English language. ANGF: Research idea, study design, data analysis, manuscript drafting. EAR: Research and manuscript drafting. AFG: Manuscript drafting and nuclear cardiology images interpretation. NEZ: Research idea, study design, manuscript drafting, supervision and mentorship.

\section{Conflicts of Interest}

The authors declare that they have no competing interests.

\section{References}

[1] Xie, M., Zhou, H., Cheng, T.O., Wang, J., Wang, X., Lu, Q., et al. (2013) Left Ventricular Apical Aneurysm Associated with Normal Coronary Arteries Following Cardiac Surgery: Echocardiographic Features and Differential Diagnosis. International Journal of Cardiology, 168, 3665-3670.

https://doi.org/10.1016/j.ijcard.2013.06.001

[2] Maron, M.S., Finley, J.J., Bos, J.M., Hauser, T.H., Manning, W.J., Haas, T.S., et al. (2008) Prevalence, Clinical Significance, and Natural History of Left Ventricular Apical Aneurysms. Circulation, 118, 1541-1549. https://doi.org/10.1161/CIRCULATIONAHA.108.781401

[3] Dwivedi, A., Freedberg, R., Donnino, R. and Vainrib, A. (2017) Geriatric Presentation of Idiopathic Left Ventricular Aneurysm. CASE: Cardiovascular Imaging Case Reports, 1, 84-87. https://doi.org/10.1016/j.case.2017.01.008

[4] Durko, A.P., Budde, R.P.J., Geleijnse, M.L. and Kappetein, A. (2017) Recognition, Assessment and Management of the Mechanical Complications of Acute Myocardial Infarction. Heart, 104, 1-8. https://doi.org/10.1136/heartjnl-2017-311473

[5] Lang, R.M., Badano, L.P., Mor-Avi, V., Afilalo, J., Armstrong, A., Ernande, L., et al. (2015) Recommendation for Cardiac Chamber Quantification by Echocardiography in Adults: An Update from the American Society of Echocardiography and the European Association of Cardiovascular Imaging. Journal of the American Society of Echocardiography, 28, 1-39.e14. https://doi.org/10.1016/j.echo.2014.10.003

[6] Valbuena-Lopez, S., Hinojar, R. and Puntmann, V.O. (2016) Cardiovascular Magnetic Resonance in Cardiology Practice: A Concise Guide to Image Acquisition and Clinical Interpretation. Revista Española de Cardiología, 69, 202-210.

https://doi.org/10.1016/j.rec.2015.11.011

[7] Tilkemeier, P.L., Bourque, J., Doukky, R., Sanghani, R. and Weinberg, R.L. (2017) ASNC Imaging Guidelines for Nuclear Cardiology Procedures: Standardized Reporting of Nuclear Cardiology Procedures. Journal of Nuclear Cardiology, 24, 2064-2128. https://doi.org/10.1007/s12350-017-1057-y

[8] Patel, N., Patel, N.J., Macon, C.J., Thakkar, B., Desal, M., Rengifo-Moreno, P., et al. (2016) Trends and Outcomes of Coronary Angiography and Percutaneous Coronary Intervention after out-of-Hospital Cardiac Arrest Associated with Ventricular Fibrillation or Pulseless Ventricular Tachycardia. JAMA Cardiology, 1, 890-899. https://doi.org/10.1001/jamacardio.2016.2860 
[9] Scanion, P.J., Faxon, D.P., Audet, A.M., Carabello, B., Dehmer, G.J., Eagle, K.A., et al. (1999) CC/AHA Guidelines for Coronary Angiography: Executive Summary and Recommendations. A Report of the American College of Cardiology/American Heart Association Task Force on Practice Guidelines (Committee on Coronary Angiography) Developed in Collaboration with the Society for Cardiac Angiography and Interventions. Circulation, 99, 2345-2357. https://doi.org/10.1161/01.CIR.99.17.2345

[10] Haberheurer, A., Andreas, M., Wiedemann, D., Rath, C. and Kacher, A. (2013) Giant Lateral Left Ventricular Wall Aneurysm Sparing the Submitral Apparatus. Journal of Cardiothoracic Surgery, 8, 201. https://doi.org/10.1186/1749-8090-8-201

[11] Flam, B. and Albåge, A. (2018) Large Inferolateral Left Ventricular Aneurysm. Annals of Cardiac Anaesthesia, 21, 68-70.

[12] Yomn, N., Houissa, K., Banaser, A., Gharbaoui, M., Bekir, O., Daly, Y., et al. (2018) Interventricular Septal Aneurysm: An Exceptional Cause of Sudden Death in Young Adult. Journal of Forensic Sciences \& Criminal Investigation, 8, Article ID: 555731. https://doi.org/10.19080/JFSCI.2018.08.555731

[13] Carcano, C., Kanne, J.P. and Kirsch, J. (2016) Interventricular Membranous Septal Aneurysm: CT and MR Manifestations. Insights Imaging, 7, 111-117. https://doi.org/10.1007/s13244-015-0456-3

[14] Kharwar, R.B., Sethi, R. and Chandra, S. (2015) Basal Interventricular Septal Aneurysm in Rheumatic Mitral Stenosis. Journal of Cardiovascular Ultrasound, 23, 52-53. https://doi.org/10.4250/jcu.2015.23.1.52

[15] Bisoyi, S., Dash, A.K., Nayak, D., Sahoo, S. and Mohapatra, R. (2016) Left Ventricular Pseudoaneurysm versus Aneurysm a Diagnosis Dilemma. Annals of Cardiac Anaesthesia, 19, 169-172. https://doi.org/10.4103/0971-9784.173042

[16] Farag, M., Lota, A., Rosendahl, U. and Roussin, I. (2019) Large Left Ventricular Apical Pseudoaneurysm: A Multimodal Imaging Approach Guiding Successful Diagnosis and Surgical Management. European Heart Journal-Case Reports, 3, ytz020. https://doi.org/10.1093/ehjcr/ytz020

[17] Frances, C., Romero, A. and Grady, D. (1998) Left Ventricular Pseudoaneurysm. Journal of the American College of Cardiology, 32, 557-561. https://doi.org/10.1016/S0735-1097(98)00290-3

[18] Abbara, S., Blanke, P., Maroules, C.D., Cheezum, M., Choi, A.D., Han, B.K., et al. (2016) SCCT Guidelines for the Performance and Acquisition of Coronary Computed Tomographic Angiography: A Report of the Society of Cardiovascular Computed Tomography Guidelines Committee Endorsed by the North American Society for Cardiovascular Imaging (NASCI). Journal of Cardiovascular Computed Tomography, 10, 435-449. https://doi.org/10.1016/j.jcct.2016.10.002

[19] Sharma, A. and Kumar, S. (2015) Overview of Left Ventricular Outpouchings on Cardiac Magnetic Resonance. Cardiovascular Diagnosis and Therapy, 5, 464-470. 


\section{Abbreviations}

CMR: Cardiac Magnetic Resonance

TTE: Transthoracic Echocardiography

NC: Nuclear Cardiology

NYHA: New York Heart Association

LVA: Left Ventricular Aneurysm

LVEF: Left Ventricular Ejection Fraction

LV: Left Ventricle

AMI: Acute Myocardial Infarction

HT: Hypertension

DM: Diabetes Mellitus 